\title{
YinQiSanHuang Jiedu decoction for the treatment of hepatitis B-related compensated liver cirrhosis: study protocol for a multicentre randomised controlled trial.
}

\section{Qing-Juan Wu}

China Academy of Traditional Chinese Medicine Guanganmen Hospital: China Academy of Chinese Medical Sciences Guanganmen Hospital

Wen-Liang Lv ( $\sim$ wenlianglv@126.com )

Guang'anmen Hospital of China Academy of Chinese Medical Sciences. https://orcid.org/0000-00024552-919X

Juan-Mei Li

China Academy of Traditional Chinese Medicine Guanganmen Hospital: China Academy of Chinese Medical Sciences Guanganmen Hospital

\section{Ting-Ting Zhang}

China Academy of Traditional Chinese Medicine Guanganmen Hospital: China Academy of Chinese Medical Sciences Guanganmen Hospital

\section{Wen-Hui Zhou}

China Academy of Traditional Chinese Medicine Guanganmen Hospital: China Academy of Chinese Medical Sciences Guanganmen Hospital

\section{Qiang Zhang}

China Academy of Traditional Chinese Medicine Guanganmen Hospital: China Academy of Chinese Medical Sciences Guanganmen Hospital

\section{Jiu-Chong Wang}

China Academy of Traditional Chinese Medicine Guanganmen Hospital: China Academy of Chinese Medical Sciences Guanganmen Hospital

\section{Qing-Nan Wang}

China Academy of Traditional Chinese Medicine Guanganmen Hospital: China Academy of Chinese Medical Sciences Guanganmen Hospital

\section{Zi-Ang Yao}

China Academy of Traditional Chinese Medicine Guanganmen Hospital: China Academy of Chinese Medical Sciences Guanganmen Hospital

\section{Rui Qiang}

China Academy of Traditional Chinese Medicine Guanganmen Hospital: China Academy of Chinese Medical Sciences Guanganmen Hospital 


\section{Si-Tong Chen}

China Academy of Traditional Chinese Medicine Guanganmen Hospital: China Academy of Chinese Medical Sciences Guanganmen Hospital

\section{Xin Zhao}

China Academy of Traditional Chinese Medicine Guanganmen Hospital: China Academy of Chinese Medical Sciences Guanganmen Hospital

\section{Shuang Liu}

China Academy of Traditional Chinese Medicine Guanganmen Hospital: China Academy of Chinese Medical Sciences Guanganmen Hospital

\section{Zheng-Min Cao}

China Academy of Traditional Chinese Medicine Guanganmen Hospital: China Academy of Chinese Medical Sciences Guanganmen Hospital

\section{Lei Xu}

China Academy of Traditional Chinese Medicine Guanganmen Hospital: China Academy of Chinese Medical Sciences Guanganmen Hospital

\section{Gao-Hui Li}

China Academy of Traditional Chinese Medicine Guanganmen Hospital: China Academy of Chinese Medical Sciences Guanganmen Hospital

\section{Jing Chen}

China Academy of Traditional Chinese Medicine Guanganmen Hospital: China Academy of Chinese Medical Sciences Guanganmen Hospital

\section{Li Wang}

China Academy of Traditional Chinese Medicine Guanganmen Hospital: China Academy of Chinese Medical Sciences Guanganmen Hospital

\section{Study protocol}

Keywords: liver cirrhosis, hepatitis B, anti-viral therapy

Posted Date: April 27th, 2021

DOI: https://doi.org/10.21203/rs.3.rs-173920/v1

License: (a) (i) This work is licensed under a Creative Commons Attribution 4.0 International License. Read Full License 


\section{Abstract}

Introduction: Hepatitis B-related compensated liver cirrhosis is related to higher risk of hepatocellular carcinoma, anti-viral therapy is the preferred method. As the pathological mechanisms of liver fibrosis are complex, drugs developed for a single target are difficult to be effective in clinical practice, so there are no chemical drugs or biological drugs with clear efficacy available for clinical application at present. Traditional Chinese medicine is a kind of medical science that has been gradually formed during thousands of years and continuously enriched by the people of all ethnic groups in China. Traditional chinese medicine shows curative effects in the treatment of liver diseases, especially in the field of liver fibrosis prevention and treatment. This study aim to test the integrative medicine (chinese medicine plus anti-riral therapy) effective on lowing hepatocellular carcinoma risk among patients with hepatitis Brelated compensated liver cirrhosis.

Methods and Analysis: This is a multicentre randomised controlled trial, total 5 hospitals and 802 patients will involved in. All the subjects are randomly allocated to the YinQiSanHuang Jiedu decoction(YQSHD) group $(n=401)$ or the placebo group $(n=401)$. The YQSHD group receives YQSHD granule with Entecavir(ETV), the placebo group receives YQSHD placebo with ETV. Treatment period will last for 52 weeks, and follow-up period for $52 \pm 2$ weeks. The primary outcome measure is the annual incidence of HCC. Outcomes will be assessed at baseline and after treatment. Objective of this trial is "the integrative of YQSHD with ETV reduce the annual incidence of HCC to 1\%".

Ethics and dissemination:The protocol has been approved by the Medical Ethics Committee of Guang'anmen Hospital, China (No.2019-006-KY), and the other centres in the trial will not begin recruiting until local ethical approval has been obtained.Trial final results will be disseminated via publication.

Trial registration: ChiCTR1900021532, this protocol was registered in the Chinese Clinical Trial Registry (URL: http://www.chictr.org.cn/searchproj.aspx) on February $26^{\text {th }}, 2019$.

\section{Background}

Hepatocellular carcinoma (HCC) is the second leading cause of cancer-related mortality worldwide, while liver cirrhosis(LC) is the main risk factor for $\mathrm{HCC}^{[1]}$. Liver fibrosis is involved in most chronic liver diseases, with further development could leading to liver cirrhosis, which affects the health and life of patients seriously ${ }^{[1]}$. A prospective study showed that the annual incidence of chronic hepatitis $B(\mathrm{CHB})$ progressing to $L C$ was $2 \% \sim 10 \%{ }^{[2]}$. Therefore, active treatment of liver fibrosis, reversing or delaying its development to improve prognosis of disease and the quality of life of patients, which has a very important significance. Nucleos(t)ide analogues (NAs) are the first-line treatment option for most patients with CHB, and the risk of developing HBV-related HCC is reduced by antiviral therapy ${ }^{[3]}$. Entecavir(ETV) is a kind of NAs recommended for HBV-related cirrhosis treatment, which is reported that it could relieve LC symptoms, improve patient prognosis and prevent the development of HCC. It was reported that long-term treatment with ETV, Lamivudine(LDV), or Tenofovir(TDF) could reverse HBV- 
related cirrhosis to milder fibrosis ${ }^{[4]}$. However, the selection of resistant mutants and nephrotoxicity during long-term therapy limit its use ${ }^{[5-6]}$. Therefore, the treatment goals for LC patient are to maximize long-term inhibition of HBV replication ${ }^{[7]}$, reducing liver cell inflammation and necrosis and liver fibrous tissue hyperplasia, delaying and reducing the occurrence of liver failure, decompensation of LC, HCC and other complications, improving the quality of life of patients, and extending their survival time, and for some patients could pursued the clinical cure ${ }^{[8-9]}$. To achieve those treatment goals, many patients and internal medicine doctors seek supplemental and alternative therapies in addition to the antiviral methods, such as traditional Chinese medicine(TCM).

In China, about $85 \%$ of HCC occurring on the basis of LC, early prevention, early diagnosis and early treatment are the keys to reducing incidence and mortality of $\mathrm{HCC}^{[10]}$. The purpose of treatment in the compensatory phase of LC is to control viral replication, inhibit disease progression, avoid complications, and reduce fibrosis. At present, there is no medicine has been clinically and effectively verified for antiliver fibrosis, but TCM has playing an important role in delaying the disease progression ${ }^{[11]}$. A study demonstrated that long-term TCM use may attenuate LC risk in patients with $\mathrm{CHB}$, the research results showed that TCM users had a significantly lower liver cirrhosis risk than TCM nonusers (adjusted HR = $0.416,95 \% \mathrm{Cl}, 0.231-0.749)$, the histological evaluation revealed improved fibrosis in $45.0 \%$ of TCM users and $11.1 \%$ of TCM nonusers $(=0.033)$. At the same time, the analysation of the prescriptions including total 119 single Chinese herbs medicinal demonstrated that "replenish qi and fortify the spleen," "clear heat and dispel dampness," and "soothe the liver and regulate qi" are the main treatment methods of TCM for $\mathrm{CHB}^{[12]}$. Alisma Shugan Decoction (ASD), a kind of traditional chinese medicine compound, ameliorates hepatotoxicity and associated liver dysfunction by inhibiting oxidative stress and p65/Nrf2/JunD signaling dysregulation in vivo ${ }^{[13]}$. Clinical study has reported that the integrative medicine therapy (ETV plus TCM) can promote the reduction of HBsAg level and the clearance of HBeAg in CHB patients with partial response to ETV through regulating the differentiation of B-cell subsets; another clinical research shows that combination therapy of traditional chinese medicine plus ETV for 48 weeks resulted in a higher rate of necro inflammatory improvement and fibrosis regression than ETV alone in CHB patients with serious liver fibrosis/cirrhosis. ${ }^{[14-15]}$ The above researches have proven that TCM plays an important role in the prevention and treatment of liver fibrosis, however, there is still lacking of large-sample, multi-center randomized controlled studies to provide reliable and high-level evidencebased medical evidence for the prevention and treatment of liver fibrosis by TCM.

In TCM theory, pathogenesis of LC is the deficiency of the essence and the deficiency of the essence, correspondingly, the main treatment principles include promoting blood circulation and removing blood stasis, strengthening the body tonic, clearing away heat, detoxifying and removing dampness. YinqiSanhuang Jiedu Decoction(YQSHD) is a traditional Chinese medicine compound, the main components are Huang Qi(Astragalus propinquus Schischkin., Radix Astragali), Yin Chen(Artemisia capillaris Thunb., Virgate Wormwood Herb Capillary Wormwood Herb), and so on, as shown in the table 1. It shows the clinical effects of "clearing away heat", "detoxifying and removing dampness", "promoting blood circulation", "removing stasis", and "strengthening the body". Which has been used clinically for 
many years, in 2018, we conducted a clinical observational study enrolled in 100 subjects. We observed two groups'(combination group oral TCM plus ETV, and control group oral ETV only) CHB-related symptoms and signs, such as losing of appetite, fatigue, flank pain, yellowing of the body, fullness of the abdominal abdomen, dry eyes, nausea, belching, dull complexion, dry mouth, bitter mouth, loose stools, and frequent nocturia, results showed that two groups were improving significantly with the extension of the treatment time. Besides, with the extension of treatment period, the alanine aminotransferase(ALT), aspartate aminotransferase(AST), total bilirubin (TBIL), and gamma-glutamyl transferase (GGT) of the two groups decreased significantly, with the combination group was dropping obviously than control group. The normalization rate of ALT in the control group was $52 \%$, and in the combination group was $94 \%$; in terms of virological response, the HBV-DNA conversion rate in the control group was $62 \%$, and the combination group was $86 \%$, those differences between the two groups are statistically significant ${ }^{16]}$. In the previous laboratory study, we had established the liver fibrosis model by intraperitoneal injection of $40 \% \mathrm{CCl}_{4}(2 \mathrm{ml} / \mathrm{kg})$ to wistar rat for 4 weeks, and extracted the three-dimensional imaging of live cells of primary rat liver sinusoidal endothelial cells(LSECs), which maximized the fidelity of the cell's in vivo life state. Through the atomic force microscope(AFM) to "palp" the cells, introduce the concept of Biopharmacology into the research. The results showed that the TCM group (intervened by astragalus polysaccharide(AP), which is the main active ingredient of the astragalus medicine in YQSHD) under the exposure mode of atomic force microscope LSECs cells spread more obviously, and the Young's modulus of LSECs after fibrotic serum injury was higher than control group(the decrease in Young's modulus means that the rigidity of the cells becomes smaller). At the same time, with the aid of a fast laser confocal fluorescence microscope/total internal reflection fluorescence microscope combined imaging system, the effect of AP on the secretion of NO in LSECs was observed. Medium and high concentrations of AP solutions can slow down the decrease in the amount of NO synthesized(NOs) in LSECs (those results have not yet been published). In order to test the effectiveness and safety of YQSHD and test its effect on delaying the progression of compensated liver fibrosis combined with ETV, we designed this multi-center, large sample randomized controlled blinded trial. The purpose of the study is to reduces the annual incidence of compensated liver cirrhosis to HCC to $1 \%$.

\section{Methods}

\section{Study setting and Recruitment}

A total 802 patients will be recruited from 10 hospitals: Guang'anmen Hospital of China Academy of Chinese Medical Sciences is the the responsible unit and will recruit 82 cases,XiXi Hospital of HangZhou will recruit 80 cases, Shuguang Hospital Affiliated to Shanghai University of traditional Chinese Medicine will recruit 80 cases, the Sixth People's Hospital of Qingdao will recruit 80 cases, Nanjing Second Hospital will recruit 80 cases, Nanchang Ninth Hospital will recruit 80 cases, BeiJing ShunYi Traditional Chinese Medicine Hospital will recruit 80 cases, the Sixth People's Hospital of ShenYang will recruit 80 cases, BeiJing DiTan Hospital Capital Medical University will recruit 80 cases, ChengDu University of Chinese Medicine Affiliated Hospital will recruit 80 cases. 
Outpatients in clinics are the main recruitment objects. Poster and online publicity with a brief introduction to the trial and the contact information of researchers will also be used for recruitment. Before enrollment, every participant will be provided with a complete and comprehensive description of the test procedure, purpose, potential adverse events and expected benefits. All subjects will be evaluated during the screening period to test whether they meet the inclusion criteria, and they will be informed that they may withdraw from the trial anytime. The screening evaluation includes: the general situation, disease-related symptoms and signs, and corresponding laboratory tests, including: urine pregnancy test (women of childbearing age), HBV DNA, HBsAg, HBsAb, HBeAg, HBeAb, HBcAb, liver function, Alphafetoprotein(AFP), liver B-ultrasound or MRI/CT and other examinations.

\section{Eligibility criteria:}

\section{Inclusion criteria}

The inclusion criteria are as follows:

a. patients have with hepatitis B-related compensatory liver cirrhosis;

b. between 18 to 65 years old;

c. patients show syndromes of liver stagnation and spleen deficiency and dampness in TCM. (For the TCM diagnostic criteria, we refer to 'National Standards for TCM Clinical Diagnosis and Treatment of the People's Republic of China' ${ }^{[17]}$ and the 'Medical Consensus of diagnosis and treatment of cirrhosis with integrated TCM and Western medicine', $\left[{ }^{18]}\right.$ which was published by Digestive System Diseases Committee, Society of Integrated Traditional Chinese and Western Medicine.);

d. voluntary signing of informed consent.

\section{Exclusion criteria}

The exclusion criteria are as follows:

a. patients with liver cirrhosis caused by other chronic liver diseases;

b. Patients with acute and chronic hepatitis, autoimmune hepatitis, primary biliary cirrhosis, primary sclerosing cholangitis, genetic metabolic liver disease, drug or toxic hepatitis, alcoholic liver disease with non-HBV hepatotropic virus infection;

c. pregnant or lactating women or women planning to become pregnant during the study period》

d. patients who are allergic to the test drugs $₫$

e. patients who have mental disorders that cannot cooperate with the study, or patients with epilepsy in unstable status $\Downarrow$

f. patients with severe systemic diseases related with heart, brain, lung, kidney, and hematopoiesis $\rrbracket$

g. patients of alcoholism or with other unsuitable conditions that not suitable for enrollment. For those patients who are already using TCM, we will not enroll them unless they have stop using TCM for more than three months; 
h. other situations deemed unsuitable by the investigator.

If the following conditions occur, the subjects should discontinue the trial: a) poor compliance, irregular taking medicine, failure to revisit or revisit on time. b) some combined diseases or complications, or deterioration during the trial. c) subject self-withdrawal. d) combined other drugs, or not taking test drugs according to research regulations. e) lost contact. f) cannot provide complete information. There are specific stopping criteria: a) serious safety problems occurred during the test, and the test should be stopped in a timely manner. b) the drug was found to have no clinical value during the trial, and the trial should be stopped to avoid delaying the effective treatment of subjects. c) it is found in the trial that there is a major error in the clinical trial protocol and it is difficult to evaluate the effect of the drug; or a welldesigned protocol with important deviations in the implementation, and it is difficult to continue to evaluate the efficacy and safety of the drug. d) the funding supporter requested stopping (such as funding reasons, management reasons, etc.). e) the State Food and Drug Administration of China ordered the trial to be stopped for some reason. $\mathrm{f}$ ) the test is suspended due to force irresistible reasons.

\section{Interventions:}

The test group (YQSHD group) receives YQSHD formula granules $5 \mathrm{~g}$ (brewed with $150-200 \mathrm{ml}$ water before taken $\bowtie$ twice a day, combined with Entecavir( H20100019, ChiaTai TianQing Pharmaceuticals in Jiangsu, China) $0.5 \mathrm{mg}$ once a day. The control group (placebo group) receives YQSH placebo formula granules $5 \mathrm{~g}$ (brewed with 150-200ml water before taken $\bigotimes$ twice a day, combined with Entecavir $0.5 \mathrm{mg}$ once a day. The main compositions of YQSHD, totally 14 kinds of herb, are shown in Table 1 . The test drugs are made into Chinese medicine formula granule. YQSHD placebo is made of excipients, thinners, coloring agents, flavoring agents and fried malt, which is similar to YQSHD in the shape, color, smell and taste.

Other antiviral medicines or TCM with similar clinical efficacy must not be taken during the trial, such as TDF or LDV, if the un-antiviral medicines combined, record them in the 'Case Report Form (CRF)'. If the subjects need other treatment or concomitant care, they should contact the doctor in advance.

\section{Outcomes:}

\section{Primary outcome}

The primary outcome is the annual incidence of HCC (the examination items include Alphafetoprotein(AFP), liver B-ultrasound test or abdominal MRI/CT imaging).

The primary outcome is evaluated before the treatment, at the 52th weeks of treatment period, and the 52 th \pm 2 weeks of follow-up period.

\section{Secondary outcomes}


The secondary outcomes include HBV-DNA negative rate, $\mathrm{HBsAg}$ negative rate, $\mathrm{HBeAg}$ seroconversion rate, liver function (ALT, AST, GGT, ALP, ALB and TBIL), spleen thickness, and the evaluation scores of patients' clinical symptoms.

These indicators are observed before the treatment, at the 26th weeks and 52th weeks of treatment period, the 26 th \pm 2 weeks and 52 th \pm 2 weeks of follow-up period.

\section{Safety outcomes}

The safety outcomes include the adverse events (AE), laboratory test (liver function, kidney function, blood routine test, urine routine test, et al), electrocardiogram (ECG), basic vital signs, and physical examination.

And the basic vital signs are body temperature $(T)$, blood pressure(BP), respiration( $R$ ) and heart rate(HR); laboratory tests include renal function tests, blood urea nitrogen (BUN), creatinine (Cr), blood, stool and urine routine tests. These biological indicators are monitored from the baseline until the end of follow-up. (Figure 2)

\section{Participant timeline}

The treatment period is 52 weeks and the follow-up period will last for $52 \pm 2$ weeks. We draw a flow diagram to make the timeline more clearly.(Figure 1)

\section{Sample size}

The aim of this study is to reduce the annual incidence of HCC from $3 \% \sim 6 \%\left[{ }^{19]}\right.$ to $1 \%$ in CHB patients. Therefore, according to the sample size estimation formula for comparison of two sample rates, the incidence of target events is less than 0.2 (or 0.3 ) or greater than 0.8 (or 0.7 ), estimation formula as follows:

$$
\mathrm{n}=\frac{\left(u_{\alpha}+u_{\beta}\right)^{2}}{2\left(\sin ^{-1} \sqrt{p_{e}}-\sin ^{-1} \sqrt{p_{c}}\right)^{2}}
$$


The $p_{\theta}$ and $p_{c}$ represent the incidence rates of test group (YQSHD group) and

placebo group (control group) respectively, the positive event rate $\left(p_{c}\right)$ in the control group is $5 \%$, while the target event rate $\left(p_{\theta}\right)$ in the test group is set to $1 \%$. Since the values of and are small, so the degree is measured in radians, $\alpha=0.05, \beta=0.10$. In this study, two-sided test was chosen, $u_{0.05}=1.96, u_{0.10}=1.282, p_{\theta}=0.05, p_{c}=0.01$. The calculated sample size of each group is approximately 334 cases, allowing for $20 \%$ attrition, therefore, the total number of patients required for this trial is $334 *(1+20 \%) * 2=802$ cases, with 401 in each group.

\section{Allocation}

\section{Sequence generation and implementation}

In this study, the central randomization system(CRS) is used to centrally control the allocation of entire randomization scheme. The randomized system mainly includes the following modules: subject screening, randomization, emergency blinding, drug formulation, drug supply management, and other functional modules. Central random principle: The researcher uses the screening module to enter some basic information of the subject (such as date of birth, gender) and obtain the subject's unique identification number (SIN). Firstly, confirm patients with the inclusion criteria, log into the CRS, input the general information of the subjects, generate the random number and fill in the electronic Case Report Form(eCRF). Secondly, drug distributors apply for the drug number from CRS according to the random number. Finally, the drug senders verify the code on the drug package with the number in the system, then the drugs been given to patients.

\section{Concealment mechanism}

The "central randomization" method was used to conceal the allocation: when researchers determined that the subjects meet criteria, the researchers log in to the central random system, enter some basic information of the subject and obtain the subject's SIN. Then, the central random system will assign subject random number and drug number according the designed blind table. In order to make the blind method effective and reduce drug loss, the random numbers are separated from the drug numbers, but the corresponding treatment plans are consistent within the system.

\section{Blinding and emergency unblinding}

This is a double-blind trial. The blinding method is set up and implemented by the Medical Statistics Center of Tianjin University of TCM. Neither the study researchers nor the subjects know the medication grouping. In the course of the trial, there is a scientific and strict management implementation system 
and feasible operation methods. All the subjects are under a standardized observation with their clinical symptoms carefully recorded. Adverse reactions are carefully observed, and 'emergency unblinding' is required for serious adverse reactions. A regular supervision, inspection and return system to ensure the implementation of double-blinding method.

Unblinding would at the end of the test to perform a statistical analysis of all the data. The outcome assessment will be blinded. When all the research data has been entered and locked, the third party participants who save the blinding codes and the researchers will jointly unblind and submit the database to the statistical analyst. When all the statistical analysis is completed, reports of statistical analysis and clinical trial summary are gonna been written by researchers.

\section{Data collection and management}

The investigator will prepare original documents for each subject who randomly entered the study, information will be recorded in the CRF. All research results (including personal data, test documents, etc.) that appear in the original medical records will be completely confidential within the scope allowed by law. Not the full name but the name initials and the random number will be shown in CRF. The content should be comprehensive and accurate, so as to record all examination results and other relevant data. The research center shall keep these documents properly for 5 years after the end of the research. The researcher will authorize the relevant regulatory agency to directly access all research-related documents.

\section{Statistics analysis plan:}

\section{Outcomes}

For the statistical analysis of the comparison of the primary outcome incidence between the two groups, we will use the $\chi 2$ test and setting $P<0.05$ (95\% confidence interval) as statistically significant; and for the secondary outcomes: where the measurement data is expressed as mean \pm standard deviation, the count data is expressed as frequency and percentage $(f, \%)$, and the frequency or percentage of the efficacy evaluation index is converted into frequency and percentage $(f, \%)$. For the comparison of the mean between the two groups, the homogeneity test is performed first. If the variances are equal, the $t$ test is used. If not discarded, the non-parametric $t$ 'test is used. The measurement data of each group before and after treatment is compared using the paired $\mathrm{t} / \mathrm{t}$ t test. The comparison of grid table count data was performed using the $\chi 2$ test, and the comparison of rank data used the rank sum test. $P<0.05$ was used as the statistical difference. The data analysis will be performed by SPSS 19.0 statistical software.

The baseline is defined as the last observation data before the first medication $\llbracket$ which included demographic characteristics and clinical baseline data『age, sex, vital signs (height, weight, temperature, heart rate, blood pressure, breathing), clinical symptom score, HBV-DNA, AFP, Entecavir treatment history, $\mathrm{CHB}$ related diseases.

\section{Analysis population and missing data}


Full Analysis Set (FAS): According to the Intentional Therapy (ITT) principle, all randomized subjects' data will enter the full analysis set. For subjects who withdraw from the study early for various reasons, the missing data will be filled by the way of last observation carry forward (LOCF).

Per-protocol Set (PPS): For those who enter the study and complete treatment and follow-up, the medication compliance is $80-120 \%$, no combined medication that affected the effectiveness evaluation during the study period, with complete evaluation index data and no major test protocol violations, their indictor data will constitute the study's Per-protocol Set.

Safety set (SS): includes those subjects who received at least once treatment after randomization.

We haven't plan to do subgroup analyses or sensitivity analyses currently.

There are principles for handling the follow up losing: a) if the subjects loss is because of adverse reactions, the data will be record in the adverse reaction statistics; b) if the loss is because of ineffectiveness, the data will be included in the efficacy statistics; c)for those patients who were effective during the treatment but could not complete the entire course, and those lost to follow-up, these data will be included in the efficacy statistics and should be analyzed intentionally.

\section{Consent, harms and $\mathrm{AE}$}

When the patient agrees to participate in the trial, the subject will sign two informed consent forms, kept by the patients and researchers respectively. For those subjects meet the criteria but with involuntary or incomplete autonomy, they can also enter the trial with the consent of ethics committee, and the informed consent will be signed by their guardian.

Any adverse medical events that occur during treatment and follow-up, regardless of whether or not there is a causal relationship with the test medicines, should be considered as an adverse event (AE) and recorded in the CRF adverse event table specified. When filling out the AE report forms, it is necessary to detailed record the occurrence, time, severity, duration, measures taken and outcomes of AE. If serious adverse events occur during the trial, emergency treatments should be taken immediately and report to the responsible researcher of the trial, the ethics committees and the China State Food and Drug Administration Safety Supervision Department within 24 hours. All the adverse events should be tracked until the adverse symptoms disappear or the researchers confirm that further follow-up is no longer needed.

If the subject has an injury that is directly related to this study during the course of treatment, and it is confirmed by the medical identification, the research team will pay the subject medical expenses; for serious $A E$ caused by drug-related injuries, the research team will give the injured subject certain compensation in accordance with relevant national laws and regulations, and the compensation costs will be borne by Guang'anmen Hospital.

\section{Monitoring and Auditing}

Page $11 / 20$ 
Composition of data monitoring committee (DMC) will monitor the trial in accordance with the corresponding standard operating procedure, which is independent from the researchers. The DMC will be allowed to evaluate the quality and integrity of the study. Before this trial start, uniform training should be conducted for all the researchers in clinical trials, which including Good Clinical Practice (GCP), research protocols, Electronic Data Capture System(EDC), central stochastic systems, and the use of scales. The DMC will assess the capabilities of research centers and collect information about institutional facilities and technical equipment. During the period of study, the DMC is responsible for verifying the clinical research records with the original records, and resolving any problems that arise during the trial. The DMC will also monitor that the research centers adheres to the research protocol, arranges the supply of research drugs, and ensures that the drugs are kept under appropriate conditions in accordance with instructions. Each center should submit the main indicators to the clinical endpoint committee to be evaluated by uniform standard. The principal investigator and authorized researcher should review, electronically sign and date the eCRF. DCM have access to interim results and make the final decision with researchers to terminate the trial.

\section{Adherence}

At the trial beginning, the researcher would emphasize the importance of compliance to the subjects, and require the subjects to bring back drug package (regardless of the remaining drugs) when they visited the research center. At the same time, we will establish online platform to make immediate contact with the patient, and contact the patients at least twice a month to learn the patient's situation and remind patients to actively return to the clinic. And for those patients who were effective during the treatment but could not complete the entire course, and those lost to follow-up, these data will be included in the efficacy statistics and should be analyzed intentionally.

Each research center will receive auditing visits every three months since the first patient is enrolled. The study will be regularly monitored by a Clinical Research Associate (CRA) in accordance with the corresponding standard operating procedure, they will help monitor whether written consent and dated informed consent forms (ICF) have been obtained from all subjects. A professional medical review would to compare the data entered in the case report form (CRF) or eCRF with the original data, to ensure the quality of the data, the clinical logic, and general medical terms for the description. The researcher will properly keep the data to protect the rights and privacy of subjects, the documents in the clinical trial shall be preserved and managed in accordance with the requirements of the GCP, and the database will be maintained by EDC. The auditing procedures if independent from the investigators.

\section{Discussion And Potential Limitations}

Studies have reported that the annual incidence of cirrhosis among CHB patients who have not received antiviral treatment is $2 \%-10 \%{ }^{[19]}$, a multi-center cohort study showed that the annual incidence of liver cancer in hepatitis $B$ virus patients taking entecavir was $1.7 \%{ }^{[3]}$. At the same time, studies reported that combined therapy is superior to conventional antiviral therapies ${ }^{[20-22]}$, which not only can enhance the 
antiviral ability, on the other hand, it also can reduce the accompanying symptoms, improve the quality of life and prolong the life of patients ${ }^{[23-24]}$. Thus, the combination therapy could become a trendy of $\mathrm{CHB}$ treatment. To facilitate high validity and reliability, a strict quality control and high-quality methodology is indispensable. To facilitate appropriate high-quality methodology and strict quality control, this protocol has been developed according to the CONSORT statement[ ${ }^{[25]}$ and SPIRIT 2013 $\left[{ }^{26}\right.$. This trial is based on the combination of the first-line antiviral drug ETV plus the TCM compound YQSHD. It is a multi-center, randomized, double-blind, placebo-controlled trial, the purpose is to drop the annual liver cancer incidence among hepatitis B-related compensated liver cirrhosis patients to $1 \%$. The results from this trial may provide evidence on the effectiveness and safety of YQSHD.

There are also some limitations to the study that should be considered. Due to restrictions in research project funds and trial period, the follow-up period couldn't be longer, it is a pity that we cannot obtain follow-up data for 3 years, 5 years or even longer. Besides, For the combination therapy, there are still some problems should be clarified, such as what's the best time for combination therapy, or, whether it can be repeated after stopping the drug. Notwithstanding these limitations, the results from this study will provide new evidence about YQSHD from a well- designed trial. In addition, this study will provide a herbal prescription for adult CHB based on the Guidelines for the diagnosis and treatment of liver fibrosis in integrative medicine practice(2019) ${ }^{[27]}$.

\section{Trial Status}

The protocol version number is 1 and was finalized in October 2018. This protocol was registered in the Chinese Clinical Trial Registry (NO. ChiCTR1900021532; URL: http://www.chictr.org.cn/searchproj.aspx). The date recruitment began on 21 October 2019. The original planning completed recruitment date is approximate December 2021, however, because of the suddenly pandemic of COVID-19 in global, which will impact our enrollment seriously and induce to a delaying end date than we excepted. If it should amend the protocol, we will communicate with the investigators Ethics Committee, trial registries and other relevant parties.

Ethics and dissemination: The protocol has been approved by the Medical Ethics Committee of Guang'anmen Hospital, China (which is the central ethical approval), and the other centres in the trial will not begin recruiting until local ethical approval has been obtained.Trial final results will be disseminated via publication.

Trial registration: ChiCTR1900021532, this protocol was registered in the Chinese Clinical Trial Registry (URL: http://www.chictr.org.cn/searchproj.aspx) on February 26 ${ }^{\text {th }}, 2019$.

\section{List Of Abbreviations}

$\mathrm{AE}$, adverse event; 
ALT, alanine aminotransferase;

AST, aspartate aminotransferase;

ALP, alkaline phosphatase;

ALB, serum albumin; TBIL, total bilirubin;

BUN, blood urea nitrogen;

CHB, chronic hepatitis B;

CRS, Central Randomization System;

CRF, Case Report Form;

CHM, Chinese Herbal Medicine;

DAAs, antiviral agents;

DMC, data monitoring committee ;

ECG, electrocardiogram;

ETV,entecavir;

FDA, Food and Drug Administration;

HBV, Hepatitis B virus;

HCC, Hepatocellular Carcinoma $\rrbracket$

LC ,liver cirrhosis;

GCP, Good Clinical Practice;

GGT, gamma glutamyl transferase;

IFN, interferon;

NAs, Nucleos(t)ide aAnalogues;

SIN,subject's unique identification number;

TCM, traditional Chinese medicine;

YQSHD, YinQiSanHuang Jiedu decoction. 


\section{Declarations}

Ethics approval and consent to participate $\triangle$ The protocol has been approved by the Medical Ethics Committee of Guang'anmen Hospital, China (which is the central ethical approva), and the other hospitals in the trial will not begin recruiting until local ethical approval has been obtained. All study participants will sign two informed consent forms, one kept by the patients and the other one kept by the researcher. The results of this study will be published in a peer reviewed journal.

Consent for publication $\varangle A c c o r d i n g$ to the terms of the informed consent, unless the subject's consent is obtained, all the subject's personal information is confidential and will not be disclosed to the public. When it is necessary, the drug supervision and administration department, ethics committee, or project funding department may consult the data of the subjects. If without permission, they will not use the subject's information for other purposes or disclose it to other groups.

Availability of data and materials: This is an Open Access article which permits others to distribute, remix, adapt, build upon this work non-commercially, and license their derivative works on different terms, provided the original work is properly cited and the use is non-commercial. Trial final results will be disseminated via publication.

Competing interests $\square$ All authors confirm that this article content has no conflict of interest.

Patient and Public Involvement statement :It was not appropriate or possible to involve patients or the public in the design, or conduct, or reporting, or dissemination plans of our research.

Funding $[T$ This project is supported by the National Key R\&D Program of China (No. 2018YFC1705700). This funding source had no role in the design of this study and will not have any role during its execution, analyses, interpretation of the data, or decision to submit results.

Authors Contributors $\square \mathrm{L}-\mathrm{WL}$ conceptualized the idea and revised it critically for intellectual content, provided professional advices. W-QJ wrote the first draft of this manuscript. All authors read and approved the final manuscript. L-JM, Z-TT, Z-Q, W-JC, W-QN, C-ZM and the other authors were involved in the conception and design, statistical advice and final approval of the manuscript, we will have ultimate authority over these activities.

Acknowledgement: We thank all the participating patients for their trust in our clinic and the study.

\section{References}

[1] Trautwein C, Friedman SL ,Schuppan D, et al. Hepatic fibrosis: Concept to treatment. J Hepato/ 2015; 62: $15-24$.

[2] Fattovich G, Bortolotti F, Donato F. Natural history of chronic hepatitis B: special emphasis on disease progression and prognostic factors. J Hepato/ 2008; 48(2): 335-352. 
[3] Kim S U, Chon Y E, Seo Y S, et al. A multi-center study of trends in hepatitis B virus-related hepatocellular carcinoma risk over time during long-term entecavir therapy. J Viral Hepat 2020; undefined: undefined. DOI: 10.1111/jvh.13384

[4] Chang TT, Liaw YF, Wu SS, et al. Long-term entecavir therapy results in the reversal of fibrosis/cirrhosis and continued histological improvement in patients with chronic hepatitis B. Hepatology 2010; 52:886-93.

[5] Sun D J, Zhu L J, Yao D H, et al. Recent progress in potential anti-hepatitis B virus agents: Structural and pharmacological perspectives. Eur J Med Chem 2018; 147:205-217.

[6] Lee S H, Cheon G J, Kim H S, et al. Tenofovir disoproxil fumarate monotherapy is superior to entecaviradefovir combination therapy in patients with suboptimal response to lamivudine-adefovir therapy for nucleoside-resistant HBV: a 96-week prospective multicenter trial. Antiviral Therapy 2018; 23:219-227.

[7] Terrault NA, Lok ASF, McMahon BJ, et al. Update on prevention, diagnosis, and treatment of chronic hepatitis B: AASLD 2018 hepatitis B guidance. Hepatology 2018; 67(4): 1560-1599.

[8] European Association for the Study of the Liver. EASL 2017 clinical practice guidelines on the management of hepatitis B virus infection. $J$ Hepatol 2017; 67(2): 370-398.

[9] Mak LY, Seto WK, Fung J, et al. Novel developments of hepatitis B: treatment goals, agents and monitoring tools. Expert Rev Clin Pharmacol 2019; 12(2): 109-120.

[10] Zhou J, Sun H, Wang Z, et al. Guidelines for diagnosisand treatment of primary liver cancer in China ( 2017 Edition). Liver Cancer 2018,7(3):235-260.

[11] Nan Y M, Kong L B. Advances in diagnosis and treatment of liver cirrhosis with integrated traditional Chinese and Western medicine. Chin J Hepatol 2018; 26(5):328-331.

[12] Hou Z, Zhang J, Zhang X, et al. Long-Term Traditional Chinese Medicine Combined with NA Antiviral Therapy on Cirrhosis Incidence in Chronic Hepatitis B Patients in the Real-World Setting: A Retrospective Study. Evid Based Complement Alternat Med 2020, 2020: 3826857.

[13] Sun YF, Pan HH, Shen SH, et al. Alisma Shugan Decoction (ASD) Ameliorates Hepatotoxicity and Associated Liver Dysfunction by Inhibiting Oxidative Stress and p65/Nrf2/JunD Signaling Dysregulation In Vivo. Med Sci Monit 2020, 26: e921738.

[14] Li M, Zhou ZH, Bao T, et al. Beneficial Effects of Bushen Formula Combined with Enticavir on Chronic Hepatitis B Patients with Suboptimal Response to Enticavir by Regulating B-Cell Differentiation. Cell Physiol Biochem 2018; 48: 633-643.

[15] Gui HL, Zhao CQ, Wang Y, et al. Histological Outcome of Fuzheng Huayu plus Entecavir Combination Therapy in Chronic Hepatitis B Patients with Significant Liver Fibrosis. J Clin Transl Hepato/ 2020; 8: 277284. 
[16] Wang D P. Clinical study of Yinqi Sanhuang Jiedu Decoction combined with Entecavir in the treatment of chronic hepatitis B. Beijing University of Chinese Medicine 2018.(China)

[17] State Bureau of Technical Supervision. National Standards for TCM Clinical Diagnosis and Treatment in the People's Republic of China: Symptoms Section. Beijing: China Standard Press. 1997;4:55.(China)

[18] Chinese Society of Integrated Traditional and Western Medicine, Digestive System Diseases Committee. Consensus on diagnosis and treatment of cirrhosis with integrated traditional Chinese and Western medicine. Chinese Journal of Integrated Traditional and Western Medicine on Digestion. 2011;19:277-279.(China)

[19] Fattovich G, Bortolotti F, Donato F. Natural history of chronic hepatitis B:special emphasis on disease progression and prognostic factors. J Hepatol 2008; 48(2):335-352.

[20] He M, Wu Y, Wang M M, et al. Meta-analysis of the clinical value of oxymatrine on sustained virological response in chronic hepatitis B. Ann Hepatol 2016; 15:482-491.

[21] Zhang L, Schuppan D. Traditional Chinese Medicine (TCM) for fibrotic liver disease: Hope and hype. Journal of Hepatology 2014; 61:166-168.

[22] Kang H, Zhao Y, Li C, et al. Integrating clinical indexes into four-diagnostic information contributes to the Traditional Chinese Medicine (TCM) syndrome diagnosis of chronic hepatitis B. Sci Rep 2015; 5:9395.

[23] Chen JX, Xu QX, Wang JH, et al. A Case of Recurrent Hepatocellular Carcinoma Acquiring Complete Remission of Target Lesion With Treatment With Traditional Chinese Medicine. Integr Cancer Ther 2017;16:597-604.

[24] Xiong X, Yang X, Liu Y, et al. Chinese herbal formulas for treating hypertension in traditional Chinese medicine: perspective of modern science. Hypertension Research Official Journal of the Japanese Society of Hypertension. 2013; 36:570-579.

[25] Moher D, Hopewell S, Schulz K F, et al. CONSORT 2010 explanation and elaboration: updated guidelines for reporting parallel group randomised trials. BMJ 2010; 340: c869.

[26] Chan A W, Tetzlaff J M,Gøtzsche P C, et al. SPIRIT 2013 explanation and elaboration: guidance for protocols of clinical trials. BMJ 2013;346: e7586.

[27] Xu L M, Liu P, Shen X Z, et al. Guidelines for Diagnosis and Treatment of Hepatic Fibrosis with in integrative medicine practice (China, 2019 Edition). Chinese Journal of Integrated Traditional Chinese and Western Medicine 2019(11).(China)

\section{Table}




\begin{tabular}{|c|c|c|c|c|c|c|c|}
\hline $\begin{array}{l}\text { Chinese } \\
\text { name }\end{array}$ & $\begin{array}{l}\text { Latin } \\
\text { name }\end{array}$ & $\begin{array}{l}\text { English } \\
\text { name }\end{array}$ & $\begin{array}{l}\text { pharmacological } \\
\text { action }\end{array}$ & $\begin{array}{l}\text { Main active } \\
\text { ingredient }\end{array}$ & $\begin{array}{l}\text { The original } \\
\text { producing } \\
\text { area }\end{array}$ & $\begin{array}{l}\text { Medicinal } \\
\text { part }\end{array}$ & $\begin{array}{c}\text { Amoun } \\
t /(\%)\end{array}$ \\
\hline monger & 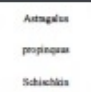 & 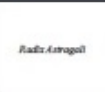 & 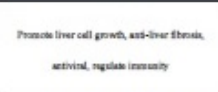 & 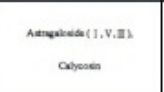 & Niamerese Cons & atives & 10:x \\
\hline mance & 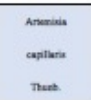 & 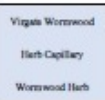 & 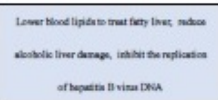 & 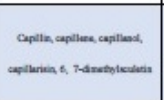 & stant Cin & 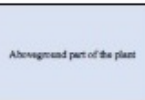 & ion \\
\hline $\operatorname{mav} 0 x$ & 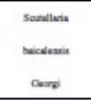 & Heves Sullap zoet & 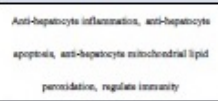 & 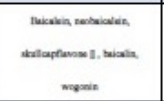 & mexcosis & atienes & 27 \\
\hline Mavelise & 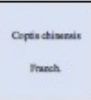 & Copte isore & 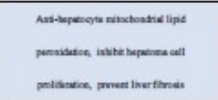 & 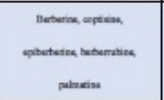 & $\cos a x \cos$ & Thereme & 27 \\
\hline Mengers & 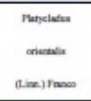 & that ef Chime & 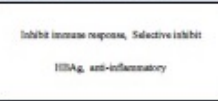 & 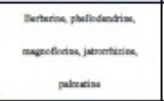 & $\operatorname{sechax} c \cos$ & Dy sost & 27 \\
\hline $\sin$ & 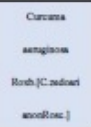 & Rabose carisum & 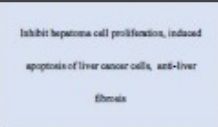 & 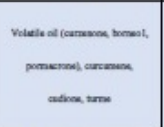 & 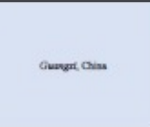 & Theser mox & s4 \\
\hline ALA & 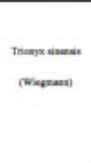 & Therte seal & 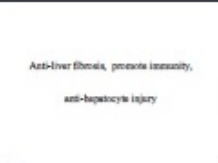 & 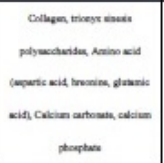 & masc coine & conpere & 27 \\
\hline Menster ate & 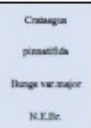 & Munson trat & 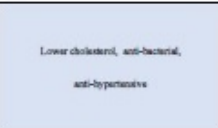 & 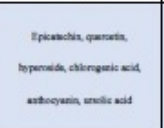 & sondene che & Viat & 120 \\
\hline sarsene & Neceuletence & 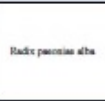 & 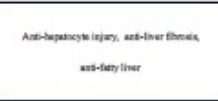 & 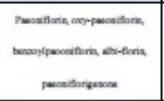 & mons ciser & nhisesen & ses \\
\hline 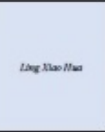 & 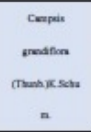 & Trenpternepe & 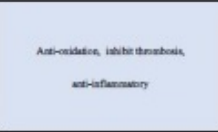 & 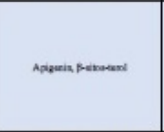 & 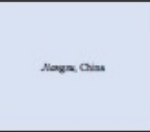 & nomer & is \\
\hline as $D_{\infty}$ & 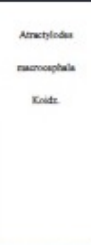 & 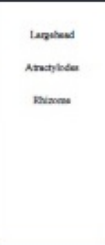 & 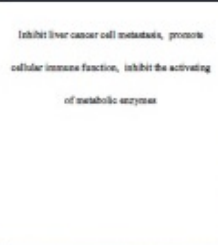 & 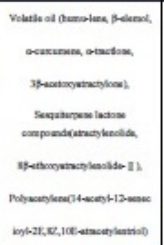 & 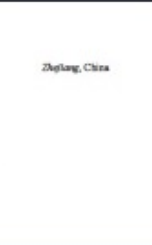 & There mext & 12 \\
\hline rative & 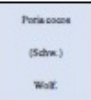 & Telater & 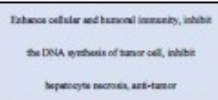 & 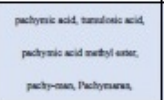 & nemen dies & Doysciente. & 12 \\
\hline charme & $\begin{array}{l}\text { Mapiense } \\
\text { chesen } \mathrm{DC} \text {. }\end{array}$ & 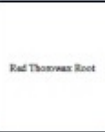 & 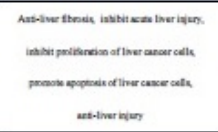 & 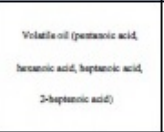 & mescosis & neinex & s4 \\
\hline 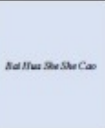 & miats & 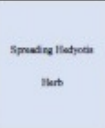 & 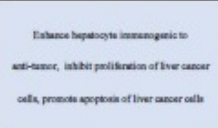 & 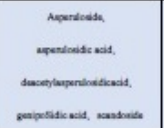 & 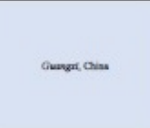 & Whoib plest & $10 \times$ \\
\hline
\end{tabular}

Figures 


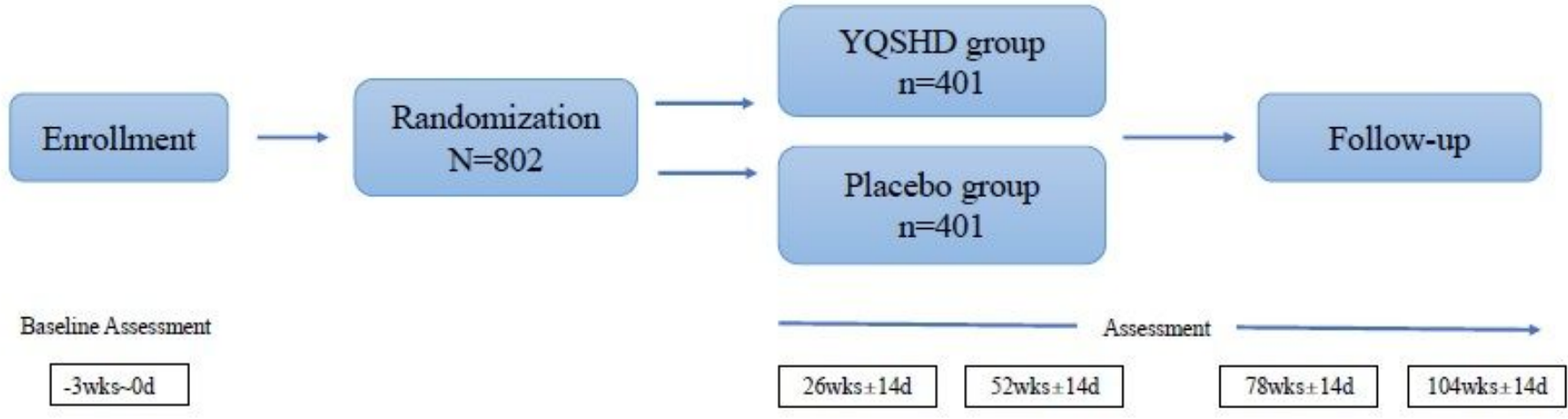

Week

\section{Figure 1}

Flow diagram of the randomized, placebo-controlled, double-blinded trial of YQSHD for hepatitis B-related compensated liver cirrhosis.

\begin{tabular}{|c|c|c|c|c|c|c|}
\hline \multirow[b]{3}{*}{ TIMEPOINT $^{\star \star}$} & \multicolumn{6}{|c|}{ STUDY PERIOD } \\
\hline & \multirow{2}{*}{$\begin{array}{c}\text { Enrolment } \\
-3 w k s-0 d\end{array}$} & \multirow{2}{*}{$\begin{array}{c}\text { Allocation } \\
\text { od }\end{array}$} & \multicolumn{2}{|c|}{ Treatment } & \multicolumn{2}{|c|}{ Follow-up } \\
\hline & & & $26 w k s \pm 14 d$ & $52 w k s \pm 14 d$ & $26 w k s \pm 14 d$ & $52 w k s \pm 14 d$ \\
\hline \multicolumn{7}{|l|}{ ENROLMENT: } \\
\hline \multirow{2}{*}{$\begin{array}{l}\text { Eligibility screen } \\
\text { Informed consent }\end{array}$} & $\mathrm{x}$ & & & & & \\
\hline & $\mathrm{x}$ & & & & & \\
\hline Allocation & & $\mathrm{x}$ & & & & \\
\hline \multicolumn{7}{|l|}{ INTERVENTIONS: } \\
\hline \multicolumn{7}{|l|}{ Placebo group } \\
\hline \multicolumn{7}{|l|}{ YQSH group } \\
\hline \multicolumn{7}{|l|}{ ASSESSMENTS: } \\
\hline $\begin{array}{r}\text { Safety } \\
\text { outcomes }\end{array}$ & $x$ & & $\mathrm{x}$ & $\mathrm{x}$ & $x$ & $x$ \\
\hline $\begin{array}{r}\text { Primary outcome: } \\
\text { annual incidence } \\
\text { of cirrhosis }\end{array}$ & & & $\mathrm{x}$ & $\mathrm{x}$ & $\mathrm{x}$ & $\mathrm{x}$ \\
\hline $\begin{array}{r}\text { Secondary } \\
\text { outcomes }\end{array}$ & & & $\mathrm{x}$ & $\mathrm{x}$ & $x$ & $\mathrm{x}$ \\
\hline
\end{tabular}


Figure 2

The schedule of enrollment, interventions and assessments demonstrated in the Standard Protocol Items: Recommendations for Interventional Trials (SPIRIT) Figure.

\section{Supplementary Files}

This is a list of supplementary files associated with this preprint. Click to download.

- informedcontentform20210106.pdf 